\title{
Geomagnetic effects on cosmic ray propagation for different conditions
}

\author{
Jimmy J. Masías-Meza ${ }^{1}$, Xavier Bertou ${ }^{1}$ and Sergio Dasso ${ }^{2}$ \\ ${ }^{1}$ Centro Atómico Bariloche (CNEA-CONICET), U.N. de Cuyo, Bariloche, Rio Negro, \\ Argentina \\ email: masiasmj@ib.cnea.gov.ar \\ email: bertou@cab.cnea.gov.ar \\ ${ }^{2}$ Instituto de Astronomía y Física del Espacio (UBA-CONICET) y Departamento de Física \\ (FCEN-UBA), Buenos Aires, Argentina \\ email: dasso@df.uba.ar
}

\begin{abstract}
The geomagnetic field $\left(B_{g e o}\right)$ sets a lower cutoff rigidity $\left(R_{c}\right)$ to the entry of cosmic particles to Earth which depends on the geomagnetic activity. From numerical simulations of the trajectory of a proton (performed with the MAGCOS code) in the $B_{g e o}$, we use backtracking to analyze particles arriving at the Auger Observatory location. We determine the asymptotic trajectories and the values of $R_{c}$ in different incidence directions. Simulations were done using several models of $B_{g e o}$ that emulate different geomagnetic conditions.
\end{abstract}

Keywords. cosmic rays, Sun: magnetic fields

\section{Introduction}

It is well known that the transport of galactic cosmic rays (GCRs) with energies $E<20 \mathrm{GeV}$ is modulated by interplanetary conditions (Ferreira et al. 2004). In addition, the trajectory of these particles in the Earth's environment can be affected by the geomagnetic field, as will be addressed in the next sections.

Since 2005, a new detection mode has been implemented in the Auger Observatory (Malargüe City). It allows the detection of transient cosmic ray effects, e.g. Forbush decreases (Abreu et al. 2011), as well as long period modulations, e.g. daily.

Using the MAGCOS code (http://cosray.unibe.ch laurent/magnetocosmics), we do numerical simulations of the trajectory of a proton and analyze the main properties of arrival at Malargüe, such as the asymptotic trajectories and the value of the rigidity cutoff $\left(R_{c}\right)$ in different incidence directions.

The International Geomagnetic Reference Field (IGRF, Finlay et al. 2010) is a semi empirical description of the Earth's magnetic field, updated every 5 years since 1955, and supported by data provided by satellites, observatories and surveys around the world. This model is mainly of dipolar topology and includes the secular variation of the main dipole moment. The Tsyganenko models (TSY, Tsyganenko 2002) include observations from major external magnetospheric sources: ring current, magnetotail current system, magnetopause currents, and large-scale system of field-aligned currents (in calm and storm conditions). In these models, the magnetosphere is deformed by the solar wind, with stretched field lines in the day-side and with a long tail in the night-side. Both models (IGRF and TSY) will be used in this study. 


\section{Cutoff rigidity calculations and asymptotic directions}

As mentioned earlier, we simulate trajectories by the backtracking method; that is, the initial location is always with Malargüe coordinates. So, we determine the "transmittance function" as follows: for each value of rigidity we put a 0 when the trajectory leads to an asymptotic direction (i.e. the incidence direction before entering to the magnetosphere), and a 1 if the particle is bent back to the Earth's surface; where case " 0 " is considered to be an allowed trajectory for the particle, and case "1" is considered a forbidden one.

The "diffuse" region between the first and last values of allowed rigidity, is called "penumbra" (Dorman 2008).

For vertical incidence (zenith $=0^{\circ}$ ), Figure 1 shows the transmittance function of protons for Malargüe city (approximate Auger Observatory location), using four different models of the geomagnetic field: Centered Dipole, Shifted Dipole, IGRF, and IGRF + TSY01 model (Tsyganenko 2002). The Shifted Dipole means that the magnetic dipole is translated from the Earth's center (Bartels 1936). In all cases, the real angle shift of the geomagnetic pole respect to the rotation axis is used. The "jump" of the penumbra from the centered dipole model to the shifted one might be produced by the loss in $\left|B_{g e o}\right|$, as the dipole shift direction on the globe is almost in the opposite direction of Malargüe's.

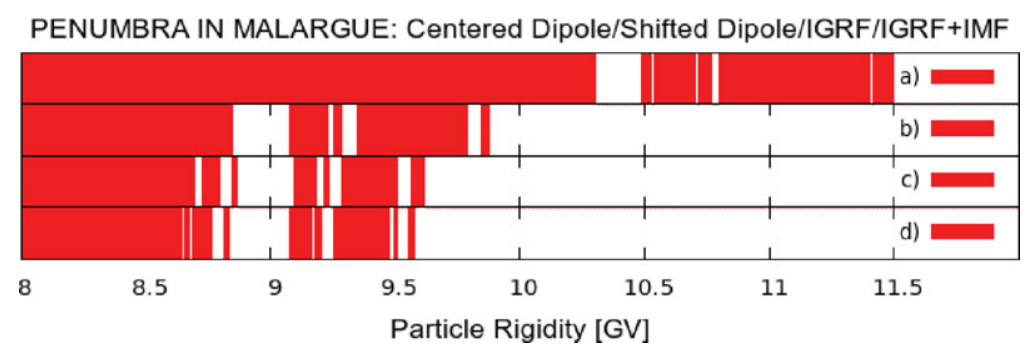

Figure 1. Transmittance function using four magnetic field models: a) Centered Dipole b) Shifted Dipole c) IGRF d) IGRF + TSY01.

The transmittance was simulated using steps of $0.001 \mathrm{GV}$ in rigidity. To obtain an effective cutoff rigidity $R_{c}$, we employ $R_{c}=R_{L}+0.001 G V * N$, where $R_{L}$ is the first low rigidity for which there is an allowed trajectory, and $N$ is the number of allowed rigidities in the penumbra region. For vertical incidence, the vertical effective cutoff rigidity at Malargüe is obtained at $R_{c}^{M l g}=9.13 \mathrm{GeV}$ (IGRF $2010+$ TSY01).
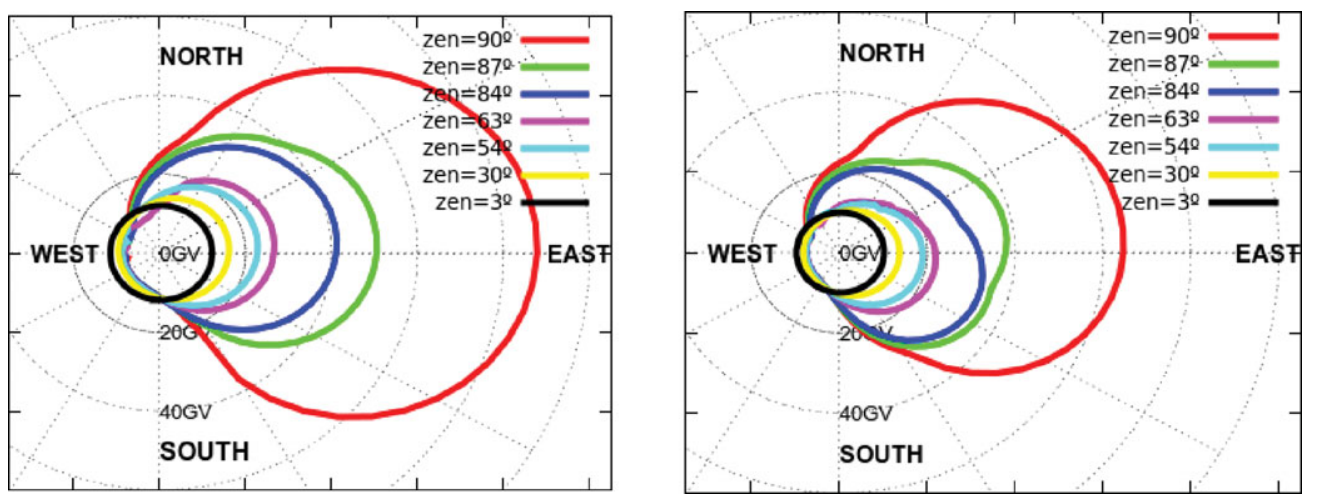

Figure 2. Effective cutoff rigidity (radius) in function of zenith (color) and azimuth (polar angle) incidence, using the Centered-Dipole model (left) and IGRF + TSY01 model (right). 
In this manner, we can obtain an effective cutoff rigidity $R_{c}$ for different zenith and azimuth incidences. This is performed by the four models, obtaining significant changes from the centered dipole to the shifted dipole. This can be seen in Figure 2, where only calculations with Centered Dipole and IGRF+TSY01 are shown.

For particle rigidities above $R_{c}^{M l g}$, asymptotic directions can be determined (Figure 3 ). We can see that as the particle rigidity decreases, the asymptotic trajectories get closer to the equator region. Computations for Rome Neutron Monitor $\left(R_{c}=6.27 \mathrm{GV}\right)$ location shows the same tendency.
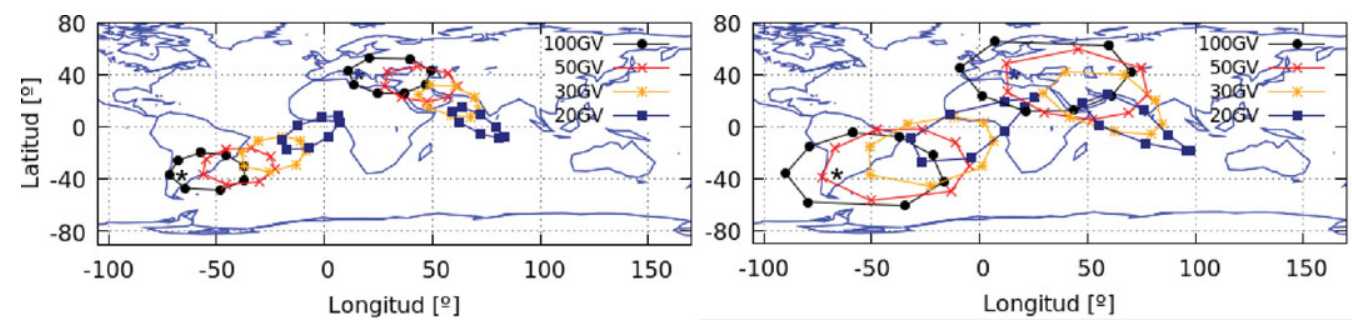

Figure 3. Asymptotic directions (projected on the Earth's surface) for two zenith incidence values: $15^{\circ}$ (left) and $30^{\circ}$ (right) and eight incidence azimuth values $\left(45^{\circ}, 90^{\circ}, \ldots, 360^{\circ}\right)$, for two locations: Auger Observatory and Rome NM, using the IGRF+TSY01 model.

\section{Simulations in geomagnetic storm conditions}

Some properties of geomagnetic disturbances can be characterized by the Dst index (see, e.g., Dasso et al. 2002). Typical values of Dst, for different levels of perturbations, are $-30 \mathrm{nT}$ (small), $-50 \mathrm{nT}$ (Moderate) and $-100 \mathrm{nT}$ (Intense), while the typical duration for these geomagnetic storms is $\sim 10 \mathrm{~h}$ (Gonzalez et al. 1994).

The values obtained for $R_{c}^{M l g}$ in different Dst conditions during a day are plotted in Figure 4. Increasing modulation for increasing $D$ st index is seen from 0nT to $-600 \mathrm{nT}$. For illustration, we evaluate the TSY01 Model, to visualize the topology of the geomagnetic field, for $D s t=0 \mathrm{nT}$ and $D s t=-250 \mathrm{nT}$ (Figure 5).

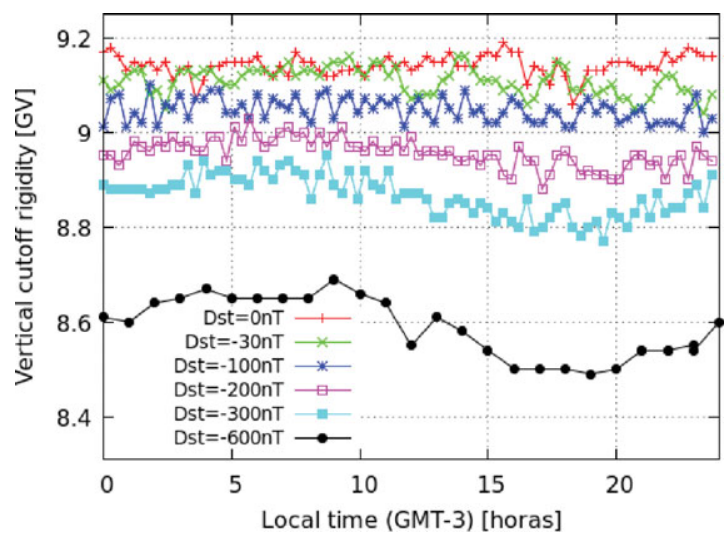

Figure 4. Effective cutoff rigidity as a function of time along the day, using the IGRF+TSY01 model, for different geomagnetic storm levels.

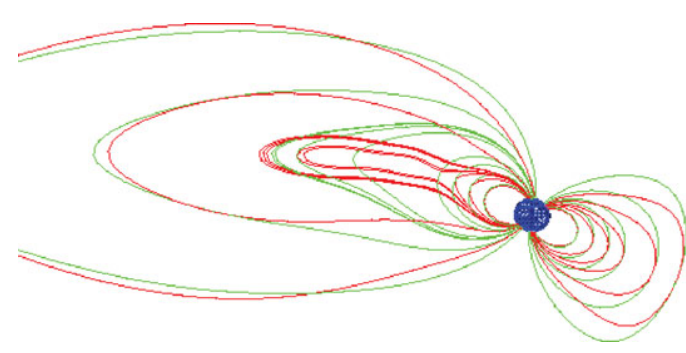

Figure 5. $B_{g e o}$ topology with $D s t=0 \mathrm{nT}$ (green) and Dst $=-250 \mathrm{nT}$ (red), with IGRF+TSY01 model. Earth size is at scale.

With the method described above, we determine the transmittance for vertical incidence into Malargüe City $\left(35.3^{\circ} \mathrm{S}, 69.3^{\circ} \mathrm{W}\right)$ for the last 20 years to see its evolution. In 
Figure 6, we show the computations for Dst $=0 \mathrm{nT}$ (calm) and $D s t=-500 \mathrm{nT}$ (very intense storm). The linear trend of the $R_{c}^{M l g}$ with time alone is $-0.03 \mathrm{GV} /$ year, while the linear trend (not shown) with $D s t$ is $-0.001 \mathrm{GV} / \mathrm{nT}$.

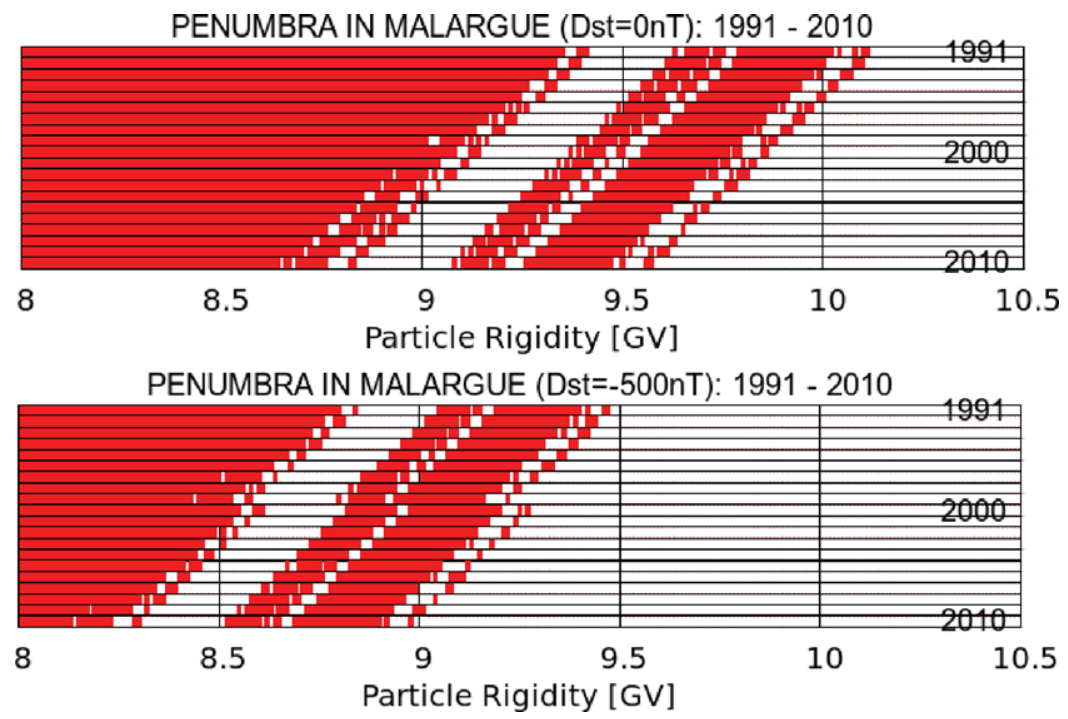

Figure 6. Transmittance functions for the last twenty years, for quiet $(D s t=0 \mathrm{nT})$ and very intense storm $(D s t=-500 \mathrm{nT})$ conditions.

\section{Conclusions}

We have determined the cutoff rigidity for different azimuth and zenith incidence directions, using trajectory computations at the site of the Pierre Auger Observatory. We identified the asymptotic direction for particles arriving at Malargüe and found that they do not change during the day. We determined the variation of the cutoff rigidity along one day for different geomagnetic storm conditions. We computed the variation of the transmittance function in the last 20 years, finding a $-0.03 \mathrm{GV} /$ year decrease in $R_{c}^{M l g}$, compatible with results in Smart \& Shea (2008). Simulations, considering different values of the $D s t$ index, show a $-0.001 \mathrm{GV} / \mathrm{nT}$ decrease of $R_{c}$ with $D s t$. All these results can be used to interpret cosmic ray modulation using Auger data for calm and storm periods.

\section{References}

Abreu, P. and the Pierre Auger collaboration 2011, JINST 6, No. P01003.

Bartels, J. 1936, J. Geophys. Res., 41, 225

Dasso, S., Gómez, D., \& Mandrini, C. H. 2002, J. Geophys. Res., 107(A5), 1059, doi:10.1029/2000JA000430

Dorman, L., Cosmic rays in magnetospheres of the Earth and other planets, Springer, 2008

Ferreira, S. E. S. \& Potgieter, M. S. 2004, Advances in Space Research, 34, 115

Finlay, C. C. et al. 2010, Geophys. J. Int., 183, 1216

Gonzalez, W. D. \& Vasyliunas, V. M. et al. 1994, J. Geophys. Res., 99, 5771

Smart, D. F. \& Shea, M. A. 2008, Proceed. of the 30th Internat. Cosmic Ray Conference, 1, 737

Tsyganenko, N. A. 2002, J. Geophys. Res., 107 (A8), doi:10.1029/2001JA000219 